\title{
Numerical Weather Prediction for Himalayan Complex Terrain: Prospects of Variational Data Assimilation
}

\author{
Sangeeta Maharjan* and Ram P. Regmi \\ National Atmospheric Resource and Environmental Research Laboratory (NARERL), \\ Central Department of Physics, Tribhuvan University, Kirtipur, Kathmandu, Nepal. \\ ${ }^{*}$ Correspondence to: sangeetamaharjan@gmail.com
}

\begin{abstract}
As part of the ongoing research activities at National Atmospheric Resource and Environmental Research Laboratory (NARERL) to realize high spatial and temporal resolution weather forecasts for Nepal, the Weather Research and Forecasting (WRF) modeling system performance with the National Center for Environmental Protection (NCEP) and National Center for Medium Range Weather Forecast (NCMRWF) initialization global meteorological data sets and the effect of surface observation data assimilation have been examined. The study shows that WRF modeling system reasonably well predicts the diurnal variation of upcoming weather events with both the data sets. The observation data assimilation from entire weather station distributed over the country may lead to the significant improvement in the accuracy and reliability of extended period of forecast. However, upper air observation data assimilation would be necessary to achieve desired precision and reliability of extended weather forecast.
\end{abstract}

Keywords: Numerical weather prediction, atmospheric modeling, data assimilation, weather disasters, complex terrain.

\section{Introduction}

Nepal covers highly diversified topographical and physiographical regions of altitudinal differences. The synoptic scale weather system in interaction with the Himalayan complex terrain undergoes dramatic modifications leading to the development of meso-scale weather systems. As a result, localized thunderstorms can be triggered which can have profound Hydro-geological and socioeconomical implications. Himalayas and its associated areas are, thus, predisposed to many weather related disasters such as floods, droughts, avalanche, windstorms, hailstorms, thunderbolts, heat and cold waves, dense fogs etc. An accurate and extended weather forecast at high spatial and temporal resolution is desired to help mitigate the weather related disaster in country.
It is unfortunate to see Nepal still practicing very conventional methodologies for forecasting weather system. It is hard to expect an accurate and reliable local weather forecast over the complex terrain of Himalayas with the adoption of conventional methods. Implementation of advanced high-resolution meso-scale meteorological models with necessary data assimilations from a good network of local meteorological observatories would be necessary to deliver need of the more precise local weather forecasts. The National Atmospheric Resource and Environmental Research Laboratory (NARERL), Central Department of Physics (CDP), Tribhuvan University, Kathmandu, Nepal has been constantly striving to realize accurate and 
extended weather predictions at high spatial and temporal resolutions aiming to enable Nepal to take effective preventive, corrective mitigation measures and reduce impacts. With this motivation, NARERL has been conducting several weather reconstruction test studies in different parts of the country [e.g., Regmi and Maharjan, 2013; Maharjan, 2014; Maharjan and Regmi, 2014; Regmi, 2014a; Regmi, 2013a; Regmi, 2013b; Maharjan and Regmi, 2013; Maharjan, 2014; Regmi, 2014b; Acharya et al., 2014] including extreme topographies of Himalayas such as Kali Gandaki valley [Regmi, 2014a] at very high $(1 \mathrm{~km}$ x $1 \mathrm{~km})$ horizontal grid and temporal (15 minutes) resolutions. Results of these studies are very encouraging and NARERL is all set to implement the realtime weather forecasting for Nepal, soon the high performance computing system and fastInternet connections are available.

However, it is important to note that the sensitivity of the meso-scale numerical weather prediction models largely dependent on the quality of initial and lateral boundary conditions provided by the global models. These initial conditions have limitations, such as coarse horizontal resolutions and inadequate representation of localized meso-scale features. In order to achieve the desired precision and reliability for extended period of forecast, it would be necessary to assimilate observational data from good network of surface and upper air measurements. It is very import to assimilate the observation data for the complex terrain of Himalayas where a myriad of extraordinary meteorological phenomena can be locally associated with the endless varieties of hills, passes, valleys and slopes.

Different assimilation techniques are available to combine available past as well as current observations of the atmosphere to best define the initial state and hence an improved analysis [Kalnay, 2003]. A brief review of different data assimilation techniques such as successive correction, optimum interpolation, and variational methods (3-DVAR, 4-DVAR) and highlights of their applications in numerical weather prediction models can be found in Navon [2009]. Incorporation of the upper air data gathered from radar, radiosonde and dropsonde measurement in the assimilation, particularly, has been found to increase the accuracy and reliability of numerical weather forecast. Moreover, different global forecasts data sets prepared by different organizations are available at different spatial and temporal resolutions to initialize regional weather forecast models. These global data may include the National Center for Environmental Protection (NCEP), USA; European Center for Medium Range Weather Forecast (ECMWRF); United Kingdom Meteorological Department (UK Met), UK and National Center for Medium Range Weather Forecast (NCMRWF), India etc. However, detail inter-comparisons of model performance with different global forecast data sets were not examined in the context of Nepal.

In this paper, we will present preliminary findings on the precision of real-time weather forecast over Himalayan complex terrain initialized with NCEP FNL and with NCMRWF global forecast data sets as well as the prospects of assimilating the surface observation data has been examined.

\section{Methodology}

In this study, we have implemented the Weather Research and Forecasting (WRF) modeling system [Skamarock et al., 2008] version 3.4 and examine its performance with the global data sets from NCEP and NCMRWF. Both the forecasts are tested against the real-time 
observation data from the surface observatory maintained and operated by NARERL which is about 500 meters away from CDP, continuously monitoring since 2012. (see Figure 1). Furthermore, the 3D-VAR data assimilation technique has been applied with the data obtained from the same observatory. Data from other observatories distributed over the country were not available for us to assimilate.

\section{a. Model configuration and initialization}

The WRF modeling system was configured for a triply nested two-way interacting mesh of horizontal grid sizes $27,9,3 \mathrm{~km}$ for coarse, fine, and finest domains, respectively, with 35 vertical levels. The top of the model calculation was set at $50 \mathrm{hPa}$. The nested domain systems and their area coverage can be seen in Figure 2. Among the physics options/schemes available in the WRF modeling system, we have used WSM 3-class simple ice, Kain-Fritsch (for coarse domain only), Unified NOAH landsurface, YSU Planetary Boundary Layer, RRTM long wave, and Dhudhia short wave etc. for this calculations. The model was separately initialized with 6 hourlymeteorological data from NCEP and NCMRWF which are approximately $110 \mathrm{~km} \times 110 \mathrm{~km}$ and $25 \mathrm{~km} \times 25 \mathrm{~km}$ horizontal resolution, respectively. In both the calculations

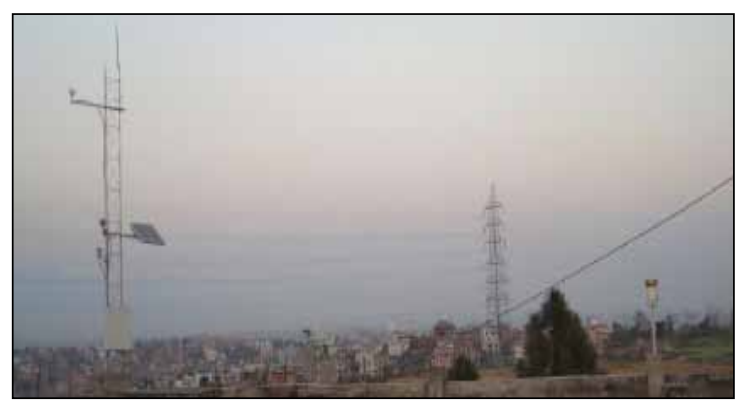

Figure 1. A picture of Automatic Weather Station located at $27.68 \mathrm{~N}, 85.28 \mathrm{E}$, maintained and operated by NARERL, Central Department of Physics, Tribhuvan University, Kirtipur at about 500 meters north from the NARERL laboratory. the 24 categories land use and 30 -second terrain elevation data by United States Geological Survey (USGS) were used.

\section{b. 3D-VAR Data Assimilations}

The data assimilation technique enable us to combine the numerical weather products (first guess or background meteorological fields) to combine with the observed meteorological fields with their error statistics to realize an improved estimates (the analysis) of the atmospheric state [Barker et al., 2004; Huang et al., 2009; Barker et al.,2012; Yang et al., 2015]. Variational (VAR) data assimilation achieves this through the iterative minimization of a prescribed cost or penalty function [Ide et al., 1997].

$$
J(x)=\frac{1}{2}\left(x-x^{b}\right)^{T} B^{-1}\left(x-x^{b}\right)+\frac{1}{2}\left(y-y^{0}\right)^{T} R^{-1}\left(y-y^{0}\right)
$$

Where $\mathrm{x}$ is the analysis to be found that minimizes the cost function $\mathrm{J}(\mathrm{x}), \mathrm{x}^{\mathrm{b}}$ is the first guess of the numerical weather prediction model, $\mathrm{y}^{0}$ is the assimilated observation, and $\mathrm{y}=\mathrm{H}(\mathrm{x})$ is the model-derived observation transformed from the analysis $\mathrm{X}$ by the observation operator $\mathrm{H}$ for comparison against $\mathrm{y}^{0}$ [Lorenc, 1966]. The solution for the cost function given by above equation represents a posteriori maximum likelihood (minimum variance) estimate of the true state given the two sources of a priori data: the first guess $\mathrm{x}^{\mathrm{b}}$ and the observation $\mathrm{y}^{0}$. The fit to individual observation points is weighted by the estimates of their errors, that is, B and $\mathrm{R}$, which are the background error covariance matrix and the observation error covariance matrix, respectively.

\section{Results}

Model was run in real-time forecasting mode for three successive days starting 


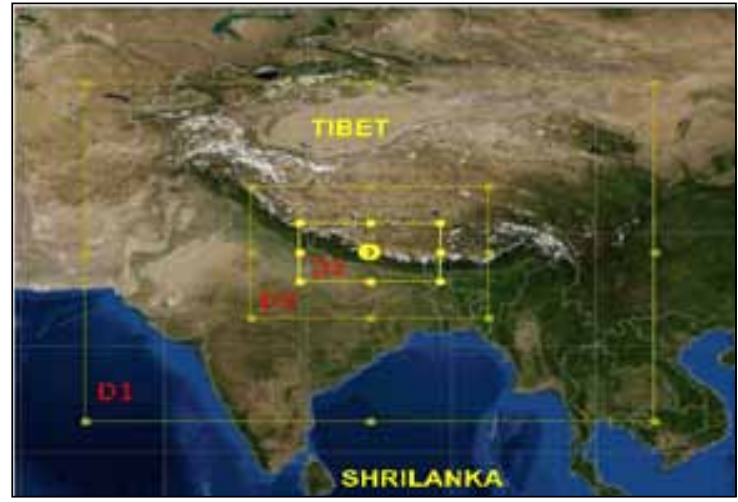

Figure 2. The triply nested domain system utilized for the present study.

from 29 May to 01 June 2014 with the same configurations with the initial global data sets from NCEP and NCMRWF in parallel. The predictions made with the two global data sets are compared with the available observed data at NARERL observatory. The effect of observed data assimilation in the numerical predictions is seen to be significant even with the single site. Present study is, thus, expected to provide an excellent starting point for further in-depth analysis in finding the best representative global data sets to initialize the WRF modeling system and assimilation of observation data to realize more precise medium range weather forecasting over the Himalayan complex terrain.

\section{a. Comparison of predictions and observations}

Figure 3a shows the composite plots of diurnal variation of predicted temperatures initialized with the two global data sets at the grid point of calculation domain within which the NARERL observatory is located. It can be understood from Figure 3a that the diurnal variation of temperature from the both the global data sets are reasonably well reproduced the observed diurnal variation of temperature (see legend, AWS-Obs.). However, the NCMRWF (see legend, WRF-NGFS) simulation appears to be relatively closer to the observation compared with the NCEP (see legend, WRF-FNL).

It should be noted that comparison has been made with the model predictions at 2 meters height above the surface whereas the observation data is at about 5 meters. Thus, a slight increase in observed temperature can be expected, at least during the daytime, if the measurements were made at 2 meters height. Since the NCMRWF predicts relatively higher temperature compared to NCEP, we expect better resemblance of observed temperature with the NCMRWF calculations.

Likewise, Figure $3 b$ shows the diurnal variation of wind speed at 10 meters height above the surface for the same period. It is clearly seen that both the global initial data
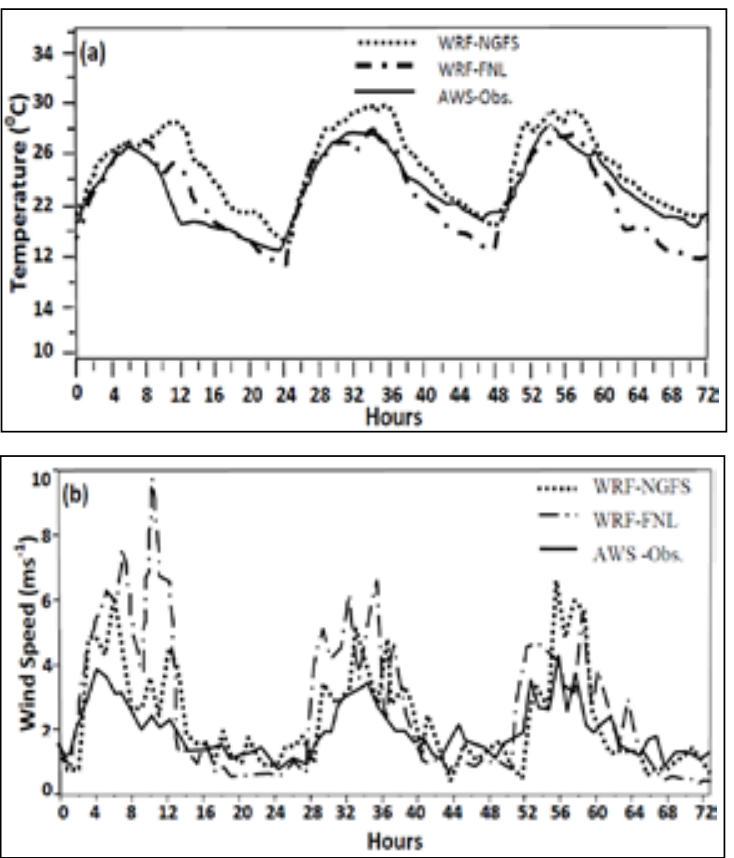

Figure 3. Comparison of forecasted and observed temperature (a) and wind speed (b) for the period of 00 UTC, 29 May to 00 UTC, 01 June 2014. Solid line is AWS observation, dotted line is WRF-NGFS and dash-dot-dash line is WRF-FNL. 
sets estimates the wind speed compared to the observation. Again, the NCMRWF gives better resembles compared with the NCEP, at least, during the daytime. In general, NCEP leads to the over estimation of wind speed.

\section{b. Sensitivity of 3D-var data assimilation}

Figure 4 shows the comparison of diurnal variation of calculated wind speed with NMCRWF global data sets with and without 3D-var data assimilation for two consecutive days. With just single station data assimilation, it is hard to expect significant change in the model prediction over the complex terrain of Himalayas. Nevertheless, it appears that the WRF model is quite sensitive even with single surface station observation data. This may imply that with the assimilation of the entire surface weather stations distributed over the whole country, significant improvements in the accuracy of model predictions can be expected. However, for desired accuracy and reliability of extended weather predictions for Nepal, assimilation of upper air observation data from Radar and Radiosonde from different parts of the country are needed.

\section{Conclusions}

The WRF model performance with NCEP and NCMRWF global initialization data sets as well as the sensitivity of the model predictions with and without data assimilation has been examined for the short period. Both the global initialization data sets reasonably well reproduced the diurnal variation of observed temperature and wind speed. The NCMRWF global data sets, perhaps, because of its high spatial resolution appears to give relatively better results compared to the NCEP data sets

The WRF model is found to be highly

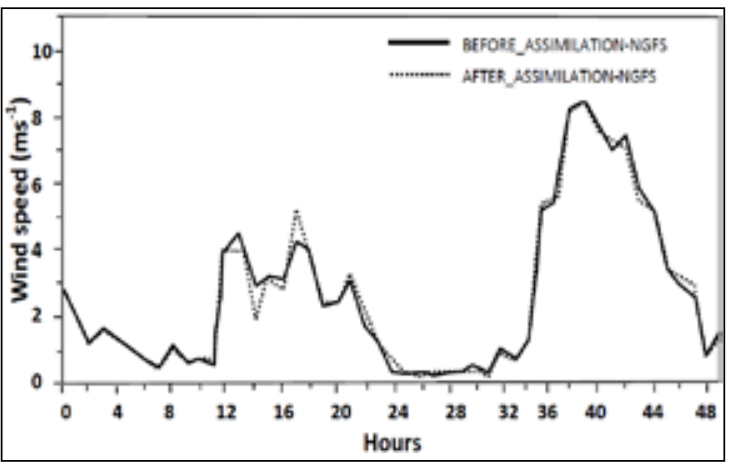

Figure 4. Two day time series plot of near surface wind at 27 meters above the surface at the grid point in which the NARERL weather station is located. Solid line represents time series without data assimilation and dotted line represent time series incorporating data assimilation.

sensitive in its prediction with the assimilation of even single observatory. It appears that good quality data assimilation from the entire surface observatories distributed over the country might leads to the significant improvement in the accuracy and reliability of extended weather predictions. However, without assimilating upper air observations in the numerical prediction, desired accuracy and reliability of extended prediction may not be possible.

\section{Acknowledgments}

This work was carried out at National Center for Medium Range Weather Forecast (NCMRWF), Noida, India using its high performance computing system. We thank Indian National Science Academy (INSA), Govt. of India for providing necessary financial support and arrangement of visit to NCMRWF in the recommendation of National Academy of Science and Technology (NAST), Nepal Government. We are very grateful to NCMRWF scientists and administration for providing every possible support, specially, Dr. S. C. Kar, Dr. S. Das and the director of NCMRWF, Dr. S. Basu. Editor N. P. Chapagain thanks the reviewers for their assistance in evaluating this paper. 


\section{References}

Acharya S., S. Neupane, R. Shrestha, C. Chapagain, P. Acharya, S. Maharjan and R. Regmi (2014), Early monsoon time local flow characteristics over Hetauda valley and its implications, Journal of Institute of Science and Technology, vol. 19, No. 1, 109-117.

Barker, D.M., W. Huang, Y.R. Guo, and Q.N. Xiao (2004), A Three-Dimensional (3DVAR) Data Assimilation System For Use With MM5: Implementation and Initial Results, Mon. Wea. Rev., 132, 897-914.

Barker, D., X.-Y. Huang, Z. Liu, T. Auligné, X. Zhang, S. Rugg, R. Ajjaji, A. Bourgeois, J. Bray, Y. Chen, M. Demirtas, Y.-R. Guo, T. Henderson, W. Huang, H.-C. Lin, J. Michalakes, S. Rizvi, and X. Zhang (2012), The Weather Research and Forecasting Mod-el's Community Variational/ Ensemble Data Assimilation System: WRFDA, Bull. Amer. Meteor. Soc., 93, 831-843.

Huang, X.Y., Q. Xiao, D.M. Barker, X. Zhang, J. Michalakes, W. Huang, T. Henderson, J. Bray, Y. Chen, Z. Ma, J. Dudhia, Y. Guo, X. Zhang, D.J. Won, H.C. Lin, and Y.H. Kuo (2009), FourDimensional Variational Data Assimilation for WRF: Formulation and Preliminary Results, Mon. Wea. Rev., 137, 299-314.

Ide, K., P. Courtier, M. Ghil, and A. C. Lorenc (1997), "Unified notation for data assimilation: operational, sequential and variational," Journal of the Meteorological Society of Japan, vol. 75, no. 1, pp. 181-189.

Kalnay, E. (2003), Atmospheric Modeling, Data Assimilation and Predictability, Cambridge University Press, London, UK.

Lorenc, A. C. (1966), "Analysis methods for numerical weather prediction," Quarterly Journal of the Royal Meteorological Society, vol. 112, no. 474, pp. 1177-1194.

Maharjan, S.(2014), Springtime meteorology of the Damauli valley, Nepal: Implications for climatic comfort and environmental hygiene, Journal of Institute of Science and Technology, vol. 19, No. 2, 24-29.

Maharjan, S. (2014), Intrusion of moisture over the Kathmandu valey from southern plain, The Himalayan Physics, Vol.5, No.5, Nov 2014.
Maharjan, S. and R. P. Regmi (2014), Grid based temperature and relative humidity distribution map of the Kathmandu valley, Journal of Institute of Science and Technology, vol. 19, No. 1, 7-13.

Maharjan, S. and R. P. Regmi (2013), Mechanism of intrusion and channeling of regional air mass over Kathmandu valley, Journal of Institute of Science and Technology, vol. 19, No. 1, 77-83.

Navon, I. M. (2009), "Data assimilation for numerical weather prediction: a review," in Data Assimilation For Atmospheric, Oceanic And Hydrologic Applications, S. K. Park and L. Xu, Eds., pp. 21-65, Springer, Berlin, Germany.

Regmi, R. P. (2014a), Aviation hazards over the Jomsom Airport of Nepal as revealed by numerical simulation of local flows. Journal of Institute of Science and Technology, vol. 19, No. 1, 111-120.

Regmi, R. P. (2014b), Aviation hazards in the sky over Thada as revealed by meso-scale meteorological modeling, Journal of Institute of Science and Technology, vol. 19, No. 2, 65-70.

Regmi, R. P. (2013a), Springtime windstorm in the Kathmandu valley and its predictability, Journal of Institute of Science and Technology, vol. 18, No. 2, 28-36.

Regmi, R. P. (2013b), Preliminary Study on the state of pre-monsoon time climatic comfort over the Chitwan valley, Nepal, The Himalayan Physics, Vol., 4, 59-69.

Regmi, R. P. and S. Maharjan (2013), Wind Energy Potential of Middle Hills of Nepal Himalaya. World Wind Energy International Quarterly Bulletin, ISSUE 1, March.

Skamarock, W. C., and Coauthors (2008), A description of the Advanced Research WRF version 3. NCAR Tech. Tep. TN-475+STR, 113 pp.

Yang, J., K. Duan, J. Wu, X. Qin, P. Shi, H. Liu, X. Xie, X. Zhang and J. Sun (2015), Effect of data assimilation using WRF-3DVAR for heavy rain prediction on the northeastern edge of the Tibetan plateau, Advances in Meteorology,Volume 2015, Article ID 294589, 14 pages http://dx.doi. org/10.1155/2015/294589 